Proyecciones Journal of Mathematics

Vol. 40, $\mathrm{N}^{\circ}$ 5, pp. 1301-1321, October 2021.

Universidad Católica del Norte

Antofagasta - Chile

\title{
The distribution of zeros of solutions for a class of third order differential equation
}

\author{
C. Cesarano \\ International Telematic University Uninettuno, Italy \\ M. A. Arahet \\ Amran University, Yemen \\ and \\ T. M. Al-shami \\ Sana'a University, Yemen \\ Received : February 2020. Accepted : April 2021
}

\begin{abstract}
For third order linear differential equations of the form $\left(r(t) x^{\prime}(t)\right)^{\prime \prime}+p(t) x^{\prime}(t)+q(t) x(t)=0$,

we will establish lower bounds for the distance between zeros of a solution and/or its derivatives. The main results will be proved by making use of Hardy's inequality, some generalizations of Opial's inequality and Boyd's inequality.
\end{abstract}

Keywords and phrases: Third order differential equations, Opial and Hardy inequalities.

2010 Mathematics Subject Classification: $34 C 10,34 K 11,34 B 05$. 


\section{Introduction}

The distribution of boundary conditions is the distribution of zeros of solutions of differential equations has been started by Picard [15, 16], who derived some uniqnueness results for solutions of the second order nonlinear differential equation with two-points boundary conditions when the nonlinear function satisfies the Lipschitz condition. The distribution of zeros of $n^{\text {th }}$ order differential equations with more than two points has been considered by Niccoletti [14]. Motivated by the work of Picard and Niccoletti. C. de la Vallee Poussin in 1929 [22] considered the general $n^{\text {th }}$ order linear differential equation

$$
y^{(n)}(t)+p_{1}(t) y^{(n-1)}(t)+\ldots+p_{n}(t) y(t)=0, t \in I,
$$

with real coefficients that are locally integrable inside $I$ and studied the disconjugacy of solutions. The equation (1.1) is said to be disconjugate on an interval $I$ if every nontrivial solution has less than $n$ zeros on $I$, multiple zeros being counted according to their multiplicity. The equation (1.1) is said to be $(k, n-k)$ - disconjugate on an interval $I$ if no nontrivial solution has a zero of order $k$ followed by a zero of order $n-k$. This means that, for every pair of points $\alpha, \beta \in I, \alpha<\beta$, there does not exist a nontrivial solution of (1.1) which satisfies

$$
\left.\begin{array}{l}
y^{(i)}(\alpha)=0, \quad i=0, \ldots, k-1, \\
y^{(j)}(\beta)=0, \quad j=0, \ldots, n-k-1 .
\end{array}\right\}
$$

The least value of $\beta$ such that there exists a nontrivial solution which satisfies (1.2), is called the $(k, n-k)$-conjugate point of $\alpha$. The first work that was published by C. de la Vallee Poussin in 1929 was on the evaluation of the length of the interval $[0, h]$ in which the boundary value problem $y\left(t_{1}\right)=y\left(t_{2}\right)=\ldots=y\left(t_{n}\right)=0,\left(0 \leq t_{1}<t_{2}<\ldots<t_{n} \leq h\right)$ for the linear differential equation (1.1) only admits the null solution. The importance of his work has been emphasized by many authors and is testified by many authors in the literature $[6,11,13,18,19,20,17,23]$. Following the way indicated by C. de la Vallee Poussin, one has tried to evaluate the length $h$ in a function of the upper bounds of the coefficients. The precise evaluation of the maximal length of the considered interval has only been obtained for second order differential equations [12] with $1 / 4$ which is the best possible constant. Coming then to the equation (1.1), C. de la Vallee Poussin proved that this equation is disconjugate in $[a, b)$, satisfies the inequality 


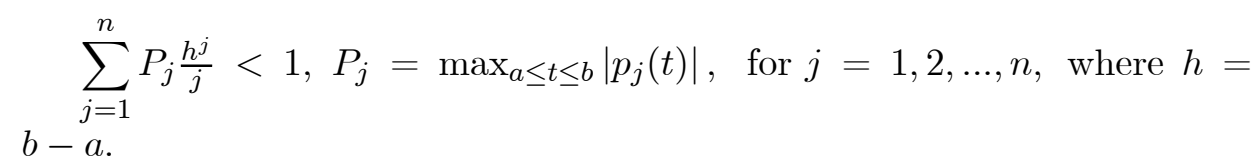

This is the part of his paper which has motivated the many refinements and generalizations described in the analysis. In this paper we interesting to study the distribution of zeros of third order differential equations. For completeness, we recall some of the related results which motivate the contents of this paper. Lasota [11] considered the third order differential equation

$$
y^{\prime \prime \prime}(t)+p_{1}(t) y^{\prime \prime}(t)+p_{2}(t) y^{\prime}(t)+p_{3}(t) y(t)=0, t \in[a, b] .
$$

and proved the following condition

$\mathrm{p}_{1} \frac{h}{4}+p_{2} \frac{h^{2}}{\pi^{2}}+p_{3} \frac{h^{3}}{2 \pi^{2}} \leq 1$,

is sufficient to make (1.3) is disconjugate on $[a, b]$ where $P_{i}=\max \left|p_{i}(t)\right|$ on $[a, b]$. Mathsen [13] proved that equation (1.3) is disconjugate on $[a, b]$ if $p_{2}(t) \leq 0$ on $[a, b]$, and

$$
\left(2 h_{1}+1\right) P_{3} \frac{\left[\exp \left(2 P_{1} h_{1}\right)-\exp \left(P_{1} h_{1}\right)-h_{1} P_{1}\right]}{P_{1}^{2}} \leq 1
$$

where $h_{1}=(b-a) / 2$. Casdei [6] also proved that if $\mathrm{P}_{1} \frac{h}{2}+P_{2} \frac{h^{2}}{8}+P_{3} \frac{h^{3}}{24} \leq 1$. then the equation (1.3) is disconjugate on $[a, b]$. Agarwal and Krishnamoorthy [1] also proved that if

$$
\mathrm{P}_{1} \frac{2 h}{3}+P_{2} \frac{h^{2}}{6}+P_{3} \frac{2 h^{3}}{81} \leq 1,
$$

then the equation (1.3) is disconjugate.

In this paper, we are concerned with the lower bounds of the distance between zeros of a nontrivial solution and/or its derivatives for the thirdorder differential equations of the form

$$
\left(r(t) x^{\prime}(t)\right)^{\prime \prime}+p(t) x^{\prime}(t)+q(t) x(t)=0, \quad t \in \mathbf{I},
$$

where $I$ is an interval of reals and $r(t), p(t)$ and $q(t)$ are real valued functions defined on $I$ such that $r(t)>0$. By a solution of (1.5) on the interval $\mathbf{J} \subseteq I$, we mean a nontrivial real-valued function $x(t) \in C^{2}(\mathbf{J})$, which has the property that $r(t) x^{\prime \prime}(t) \in C^{1}(\mathbf{J})$ and satisfies equation (1.5) on $\mathbf{J}$. The nontrivial solution $x(t)$ of $(1.5)$ is said to oscillate or to be oscillatory, if it has arbitrarily large zeros. Equation (1.5) is oscillatory if one of its nontrivial solutions is oscillatory. An equation of the form (1.5) is said to 
be disconjugate on an interval $I$ if no nontrivial solution has more than two zeros on $I$ counting multiplicities. We say that (1.5) is right disfocal (left disfocal) on the interval $[a, b],(a<b)$ if the solutions of (1.5) such that $x^{\prime}(a)=0, x(a) \neq 0\left(x^{\prime}(b)=0, x(b) \neq 0\right)$ do not have two zeros counting muliplicities in $(a, b]([a, b))$.

Following this trend, in this paper, we are concerned with the following problems for the equation (1.5):

(i) Obtain lower bounds for the spacing $\beta-\alpha$, where $x(t)$ is a nontrivial solution of (1.5) which satisfies $x(\alpha)=x^{\prime}(\alpha)=x^{\prime}(\beta)=0$, or $x(\beta)=x^{\prime}(\beta)=x^{\prime}(\alpha)=0$,

(ii) Obtain lower bounds for the spacing $\beta-\alpha$,where $x(t)$ is a non-trivial solution of (1.5) which satisfies $x^{\prime}(\alpha)=x^{\prime}(\beta)=x^{\prime \prime}(\beta)=0$, or $x^{\prime}(\beta)=x^{\prime}(\alpha)=x^{\prime \prime}(\alpha)=0$.

The paper is organized as follows: In Sections 2, we state Hardy's inequality, the extensions of Opial's inequality and Wirtinger's inequality that will be used to prove our main resluts. In Sections 3, we prove the main results for the equation (1.5) subject to the boundary conditions stated in (i) - (ii) by applying Hardy's, extensions of Opial's inequality and some new inequalities which we will state letters.

\section{Hardy, Opial and Wirtinger Inequalities}

In this section, we present the Hardy's inequality, some generalizations of Opial's inequality and Wirtinger inequalities that will be needed in the proof of the main results in Section 3. These inequalities are adapted from Agarwal and Pang [2], Beesack [3], Clark and Hinton [7], Fink [8] and Kufner et al. [9, 10].

We begin with Hardy's inequality which states that: If $y$ is absolutely continuous on $(\alpha, \beta)$, such that $y(\alpha)=0$ or $(y(\beta)=0)$, then

$$
\left(\int_{\alpha}^{\beta} p(t)|y(t)|^{n} d t\right)^{\frac{1}{n}} \leq C\left(\int_{\alpha}^{\beta} r(t)\left|y^{\prime}(t)\right|^{m} d t\right)^{\frac{1}{m}},
$$

where the weighted functions $p, r$ are measurable positive functions defined on the interval $(\alpha, \beta)$ and $n, m$ are real parameters that satisfy $0<n \leq$ $\infty$ and $1 \leq m \leq \infty$. The constant $C$ is given by 


$$
C \leq m^{\frac{1}{m}}\left(m_{1}\right)^{\frac{1}{m_{1}}} A(\alpha, \beta), \quad \text { for } 1<m \leq n, m_{1}=\frac{m}{m-1}
$$

where

$$
\begin{gathered}
\underset{A(\alpha, \beta)}{A}:=\sup _{\alpha \leq t \leq \beta}\left(\int_{t}^{\beta} p(t) d t\right)^{\frac{1}{n}}\left(\int_{\alpha}^{t} r^{1-m_{1}}(s) d s\right)^{\frac{1}{m_{1}}}, \text { if } y(\alpha)=0 \\
A(\alpha, \beta):=\sup _{\alpha \leq t \leq \beta}\left(\int_{\alpha}^{t} p(t) d t\right)^{\frac{1}{n}}\left(\int_{t}^{\beta} r^{1-m_{1}}(s) d s\right)^{\frac{1}{m_{1}}}, \text { if } y(\beta)=0 .
\end{gathered}
$$

Note that the inequality (2.1) has an immediate application to the case when $y(\alpha)=y(\beta)=0$. In this case, the inequality (2.1) is satisfied if and only if

$$
\begin{gathered}
. A(\alpha, \beta):=(c, d) \subset(\alpha, \beta) \sup \left(\int_{c}^{d} p(t) d t\right)^{\frac{1}{n}} \\
\min \left\{\left(\int_{\alpha}^{c} r^{1-m_{1}}(s) d s\right)^{\frac{1}{n_{1}}},\left(\int_{d}^{\beta} r^{1-m_{1}}(s) d s\right)^{\frac{1}{m_{1}}}\right\},
\end{gathered}
$$

exists and finite. The Beesack's inequality states that: If $y(t)$ is absolutely continuous on $[\alpha, \beta]$ with $y(\alpha)=0$, then the following inequality holds

$$
\int_{\alpha}^{\beta} q(t)|y(t)|^{m}\left|y^{\prime}(t)\right|^{n} d t \leq K_{1}(m, n) \int_{\alpha}^{\beta} p(t)\left|y^{\prime}(t)\right|^{m+n} d t
$$

where $m, n$ are real numbers such that $m n>0, m+n>1, q(t)$ and $p(t)$ are nonnegative, measurable functions defined on $(\alpha, \beta)$ such that $\int_{\alpha}^{t}(p(s))^{\frac{-1}{m+n-1}} d s<\infty$, and

$K_{1}(m, n):=\left(\frac{n}{m+n}\right)^{\frac{n}{m+n}}\left[\int_{\alpha}^{\beta} q^{\frac{m+n}{n}}(t) p^{-\frac{n}{m}}(t)\left(\int_{\alpha}^{t}(p(s))^{\frac{-1}{m+n-1}} d s\right)^{m+n-1} d t\right]^{\frac{m}{m+n}}$.

If instead $y(\beta)=0$, then $(2.5)$ holds, where $K_{1}(m, n)$ is replaced by

$K_{2}(m, n):=\left(\frac{n}{m+n}\right)^{\frac{n}{m+n}}\left[\int_{\alpha}^{\beta} q^{\frac{m+n}{n}}(t) p^{-\frac{n}{m}}(t)\left(\int_{t}^{\beta}(p(s))^{\frac{-1}{m+n-1}} d s\right)^{m+n-1} d t\right]^{\frac{m}{m+n}}$. 
The Agarwal and Pang inequality states that: If $y(t) \in C^{(n-1)}[\alpha, \beta]$ be such that $y^{(i)}(\alpha)=0,0 \leq k \leq i \leq n-1(n \geq 1), y^{(n-1)}(t)$ is absolutely continuous on $(\alpha, \beta)$, then

$$
\int_{\alpha}^{\beta} \phi(t)\left|y^{(k)}(t)\right|^{l}\left|y^{(n)}(t)\right|^{m} d t \leq H_{1}\left[\int_{\alpha}^{\beta} \varphi(t)\left|y^{(n)}(t)\right|^{c} d t\right]^{(l+m) c}
$$

where $\phi$ and $\varphi$ are nonnegative and measurable function defined on the interval $(\alpha, \beta), m, l$ are real numbers such that $c / m>1$,

$$
H_{1}:=\frac{\left(\frac{m}{m+l}\right)^{\frac{m}{c}}}{(n-k-1) !}\left[\int_{\alpha}^{\beta}\left(\phi^{c}(t) \varphi^{-m}(t)\right)^{1(c-m)}\left(G_{1, k}(t)\right)^{l(c-1)(c-m)} d t\right]^{\frac{c-m}{c}},
$$

and

$$
\mathrm{G}_{1, k}(t):=\int_{\alpha}^{t}(t-s)^{(n-k-1) c(c-1)}(\varphi(s))^{-1(c-1)} d s .
$$

If instead $y^{(i)}(\beta)=0,0 \leq k \leq i \leq n-1(n \geq 1)$, then (2.8) holds where $H_{1}$ is replaced by

$$
H_{2}:=\frac{\left(\frac{m}{m+l}\right)^{\frac{m}{c}}}{(n-k-1) !}\left[\int_{\alpha}^{\beta}\left(\phi^{c}(t) \varphi^{-m}(t)\right)^{1(c-m)}\left(G_{2, k}(t)\right)^{l(c-1)(c-m)} d t\right]^{\frac{c-m}{c}},
$$

and

$\mathrm{G}_{2, k}(t):=\int_{t}^{\beta}(s-t)^{(n-k-1) c(c-1)}(\varphi(s))^{-1(c-1)} d s$.

We also need the following inequality which is the special case of an inequality proved by Agarwal and Pang [2] with two functions. This inequality states that: If $y(t) \in C^{n-1}[\alpha, \beta]$ such that $y^{(i)}(\alpha)=0, k \leq i \leq$ $n-1,0 \leq k \leq n-1(n \geq 1)$ fixed and $y^{(n-1)}$ is absolutely continuous on $(\alpha, \beta)$, then

$$
\int_{\alpha}^{\beta} c(t)\left|y^{(k)}(t)\right|\left|y^{(k+1)}(t)\right| d t \leq C_{\alpha} \int_{\alpha}^{\beta} r(t)\left|y^{(n)}(t)\right|^{2} d t
$$

where $c(t), r(t)$ be non-negative measurable functions defined on $(\alpha, \beta)$ and

$$
C_{\alpha}:=\frac{1}{2((n-k-1) !)^{2}} \max _{t \in[\alpha, \beta]} c(t) \int_{\alpha}^{\beta} \frac{(s-\alpha)^{2(n-k-1)}}{r(s)} d s .
$$

If $y^{(i)}(\beta)=0, k \leq i \leq n-1$, then (2.11) holds where $C_{\alpha}$ is replaced by 


$$
C_{\beta}:=\frac{1}{2((n-k-1) !)^{2}} \max _{t \in[\alpha, \beta]} c(t) \int_{\alpha}^{\beta} \frac{(\beta-s)^{2(n-k-1)}}{r(s)} d s .
$$

The Wirtinger inequality due to Agarwal and Pang [2] that will also be needed states that: If $y(\alpha)=y(\beta)=0$, then

$$
\int_{\alpha}^{\beta} y^{\gamma+1}(t) d t \leq \frac{(\beta-\alpha)^{\gamma+1} \Gamma^{2}((\gamma+2) 2)}{2 \Gamma(\gamma+2)} \int_{\alpha}^{\beta}\left(y^{\prime}(t)\right)^{\gamma+1}(t) d t
$$

The Clark and Hinton inequality [7] states that: If $y \in C^{2}[\alpha, \beta]$, where $y(\alpha)=y^{\prime}(\alpha)=0$, and $1 \leq r \leq 2$, then

$$
\left(\int_{\alpha}^{\beta}|y(t)|^{2}\left|y^{\prime \prime}(t)\right|^{2} d t\right)^{\frac{1}{r}} \leq(\beta-\alpha)^{1+\frac{1}{r}} C_{2}(r) \int_{\alpha}^{\beta}\left|y^{\prime \prime}(t)\right|^{2} d t
$$

where $C_{2}(r)$

$$
C_{2}(r):= \begin{cases}((2-r) / 2(r+1))^{\frac{2-r}{\sqrt{3}}} / \sqrt{3}, \text { if } \quad 1 \leq r<2, \\ \frac{1}{\sqrt{3}}, & \text { if } r=2 .\end{cases}
$$

The Fink inequality [8] states that: If $y^{(i)}(\alpha)=0,0 \leq i \leq n-1, \mu \geq 1$, $\frac{1}{\mu}+\frac{1}{\nu}=1$, then

$$
\begin{aligned}
& \int_{\alpha}^{\beta}\left|y^{(k)}(t) y^{(r)}(t)\right| d t \leq C(n, k, r, \mu)(\beta-\alpha)^{2 n-k-r+1-2 \mu}\left(\int_{\alpha}^{\beta}\left|y^{(n)}(t)\right|^{\mu} d t\right)^{\frac{2}{\mu}}, \\
& (2.17) \\
& \text { where } 0 \leq k<r<n, \quad(n \geq 2) \text {, and } \\
& \qquad \mathrm{C}(\mathrm{n}, \mathrm{k}, \mathrm{r}, \mu)=\frac{1}{2((n-k-1) !)^{2}[(n-k-1) \nu+1]^{2 \nu}}
\end{aligned}
$$

\section{Main Results}

In this section we state and prove the main results. For simplicity, we utilize the following notations:

$$
\left.\begin{array}{c}
H_{1}\left(\alpha, \beta, G_{1,0}\right)=\frac{1}{\sqrt{2}}\left[\int_{\alpha}^{\beta} Q_{1}^{2}(t) \frac{G_{1,0}(t)}{r(t)} d t\right]^{\frac{1}{2}}, \\
G_{1,0}(t)=\int_{\alpha}^{t} \frac{(t-s)^{2}}{r(s)} d s, 1(t)=\int_{\alpha}^{t} q(s) d s,
\end{array}\right\}
$$




$$
\left.\begin{array}{c}
H_{2}\left(\alpha, \beta, G_{2,0}\right)=\frac{1}{\sqrt{2}}\left[\int_{\alpha}^{\beta} Q_{2}^{2}(t) \frac{G_{2,0}(t)}{r(t)} d t\right]^{\frac{1}{2}}, \\
G_{2,0}(t)=\int_{t}^{\beta} \frac{(t-s)^{2}}{r(s)} d s, Q_{2}(t)=\int_{t}^{\beta} q(s) d s,
\end{array}\right\}
$$

$$
A_{i}(\alpha, \beta)=J_{i}(t) \min \left\{\left(\int_{\alpha}^{c} \frac{1}{r(s)} d s\right)^{\frac{1}{2}},\left(\int_{c}^{\beta} \frac{1}{r(s)} d s\right)^{\frac{1}{2}}\right\} \text {, }
$$

where $J_{i}(t)=\sup _{(c, d) \subset(\alpha, \beta)}\left(\int_{\alpha}^{\beta} R_{i}(t) d t\right)^{\frac{1}{2}}$ and $\left|R_{i}(t)\right|=\left|Q_{i}(t)\right|+|p(t)|, i=1,2$,

$$
\begin{gathered}
{ }_{1}(\alpha, \beta):=\alpha \leq t \leq \beta \sup \left(\int_{t}^{\beta} p(t) d t\right)^{\frac{1}{2}}\left(\int_{\alpha}^{t} \frac{1}{r(s)} d s\right)^{\frac{1}{2}}, \\
C_{\alpha}:=\frac{1}{2} \alpha \leq t \leq \beta \max q(t) \int_{\alpha}^{\beta} \frac{(s-\alpha)^{2}}{r(s)} d s,
\end{gathered}
$$

$$
\begin{gathered}
{ }_{2}(\alpha, \beta):=\alpha \leq t \leq \beta \sup \left(\int_{\alpha}^{t} p(t) d t\right)^{\frac{1}{2}}\left(\int_{t}^{\beta} \frac{1}{r(s)} d s\right)^{\frac{1}{2}}, \\
C_{\beta}:=\frac{1}{2} \alpha \leq t \leq \beta \max q(t) \int_{\alpha}^{\beta} \frac{(\beta-s)^{2}}{r(s)} d s,
\end{gathered}
$$

and

$$
\psi(\alpha, \beta):=\sup _{\alpha \leq t \leq \beta}\left(\left(\int_{t}^{\beta} r(t) d t\right)^{\frac{1}{2}}\left(\int_{\alpha}^{t} \frac{1}{r(s)} d s\right)^{\frac{1}{2}}\right) .
$$

Now, we are ready to prove the following results.

Theorem 3.1. Assume that $x(t)$ is a nontrivial solution of (1.5).

(1) If $x(\alpha)=x^{\prime}(\alpha)=x^{\prime}(\beta)=0$, then

$$
4 A_{1}^{2}(\alpha, \beta)+H_{1}\left(\alpha, \beta, G_{1,0}\right)+M_{1}\left(\alpha, \beta, G_{1,1}\right) \geq 1
$$


(2) If $x^{\prime}(\alpha)=x(\beta)=x^{\prime}(\beta)=0$, then

$$
H_{2}\left(\alpha, \beta, G_{2,0}\right)+4 A_{1}^{2}(\alpha, \beta)+M_{1}\left(\alpha, \beta, G_{2,1}\right) \geq 1 .
$$

Proof. To prove (3.9), we multiply (1.5) by $x^{\prime}(t)$ and integrating from $\alpha$ to $\beta$, we obtain

$$
\int_{\alpha}^{\beta} x^{\prime}(t)\left(r(t)\left(x^{\prime}(t)\right)\right)^{\prime \prime} d t=-\int_{\alpha}^{\beta} p(t)\left(x^{\prime}(t)\right)^{2} d t-\int_{\alpha}^{\beta} q(t) x(t) x^{\prime}(t) d t .
$$

We get the following by integrating the left hand side by parts

$$
\begin{aligned}
\int_{\alpha}^{\beta} x^{\prime}(t)\left(r(t)\left(x^{\prime}(t)\right)\right)^{\prime \prime} d t & =\left.x^{\prime}(t)\left(r(t)\left(x^{\prime}(t)\right)\right)^{\prime}\right|_{\alpha} ^{\beta}-\int_{\alpha}^{\beta} r^{\prime}(t) x^{\prime}(t) x^{\prime \prime}(t) d t \\
& -\int_{\alpha}^{\beta} r(t)\left(x^{\prime \prime}(t)\right)^{2} d t
\end{aligned}
$$
find

Using the assumptions that $x^{\prime}(\alpha)=x^{\prime}(\beta)=0$ and $Q_{1}(t)=\int_{\alpha}^{t} q(s) d s$,we

$$
\begin{aligned}
\int_{\gamma_{\alpha}}^{\beta} r(t)\left(x^{\prime \prime}(t)\right)^{2} d t & =\int_{\alpha}^{\beta} p(t)\left(x^{\prime}(t)\right)^{2} d t+\int_{\alpha}^{\beta} Q_{1}^{\prime}(t) x(t) x^{\prime}(t) d t \\
& -\int_{\alpha}^{\beta} r^{\prime}(t) x^{\prime}(t) x^{\prime \prime}(t) d t
\end{aligned}
$$

Integrating the term $\int_{\alpha}^{\beta} Q_{1}^{\prime}(t) x(t) x^{\prime}(t) d t$ by parts and using the assumption that $x^{\prime}(\alpha)=x^{\prime}(\beta)=0$, we obtain

$$
\begin{gathered}
\int_{\alpha}^{\beta} Q_{1}^{\prime}(t) x(t) x^{\prime}(t) d t=\left.Q_{1}^{\prime}(t) x(t) x^{\prime}(t)\right|_{\alpha} ^{\beta}-\int_{\alpha}^{\beta} Q_{1}(t)\left(x^{\prime}(t)\right)^{2} d t-\int_{\alpha}^{\beta} Q_{1}(t) x(t) x^{\prime \prime}(t) d t, \\
=-\int_{\alpha}^{\beta} Q_{1}(t)\left(x^{\prime}(t)\right)^{2} d t-\int_{\alpha}^{\beta} Q_{1}(t) x(t) x^{\prime \prime}(t) d t .
\end{gathered}
$$

Substituting (3.12) into (3.11), we get

$$
\begin{aligned}
\int_{\alpha}^{\beta}|r(t)|\left|x^{\prime \prime}(t)\right|^{2} d t & \leq \int_{\alpha}^{\beta}\left|R_{1}(t)\right|\left|x^{\prime}(t)\right|^{2} d t+\int_{\alpha}^{\beta}\left|Q_{1}(t)\right||x(t)|\left|x^{\prime \prime}(t)\right| d t \\
& +\int_{\alpha}^{\beta}|u(t)|\left|x^{\prime}(t)\right|\left|x^{\prime \prime}(t)\right| d t .
\end{aligned}
$$

where $\left|R_{1}(t)\right|=\left|Q_{1}(t)\right|+|p(t)|$ and $u(t)=r^{\prime}(t)$. Applying the inequality (2.8) on the integral

$$
\int_{\alpha}^{\beta}\left|Q_{1}(t)\right||x(t)|\left|x^{\prime \prime}(t)\right| d t
$$


with $\phi(t)=\left|Q_{1}(t)\right|, \varphi(t)=r(t), k=0, m=l=1, n=2, c=2$ and $x(\alpha)=x^{\prime}(\alpha)=0$, we get

$$
\int_{\alpha}^{\beta}\left|Q_{1}(t)\right||x(t)|\left|x^{\prime \prime}(t)\right| d t, \leq H_{1}\left(\alpha, \beta, G_{1,0}\right) \int_{\alpha}^{\beta}|r(t)|\left|x^{\prime \prime}(t)^{2}\right| d t
$$

where $H_{1}\left(\beta, \alpha, G_{1,0}\right)$ is defined as in (3.1). Applying the inequality (2.1) on the integral $\int_{\alpha}^{\beta}\left|R_{1}(t)\right|\left|x^{\prime}(t)^{2}\right| d t$ with $y(t)=x^{\prime}(t)$ and $y(\alpha)=y(\beta)=0$, we have that

$$
\int_{\alpha}^{\beta}\left|R_{1}(t)\right|\left|x^{\prime}(t)^{2}\right| d t \leq 4 A_{1}^{2}(\alpha, \beta) \int_{\alpha}^{\beta}|r(t)|\left|x^{\prime \prime}(t)^{2}\right| d t,
$$

where $A_{1}(\alpha, \beta)$ is defined as in (3.5). Applying the inequality (2.8) on the integral

$$
\int_{\alpha}^{\beta}|u(t)|\left|x^{\prime}(t)\right|\left|x^{\prime \prime}(t)\right| d t
$$

with $y(t)=x^{\prime}(t)$ and $y(\alpha)=y(\beta)=0$, with $\phi(t)=|u(t)|, \varphi(t)=|r(t)|$, $k=1, m=l=1, n=2, c=2$ and $x(\alpha)=x^{\prime}(\alpha)=0$, we get

$$
\int_{\alpha}^{\beta}|u(t)|\left|x^{\prime}(t)\right|\left|x^{\prime \prime}(t)\right| d t \leq Z_{1}\left(\alpha, \beta, G_{1,1}\right) \int_{\alpha}^{\beta}|r(t)|\left|x^{\prime \prime}(t)^{2}\right| d t
$$

where $Z_{1}\left(\alpha, \beta, G_{1,1}\right)$ is defined in (3.3).

Substituting (3.15), (3.14) and (3.16) into (3.13). We get

$$
\begin{aligned}
\int_{\alpha}^{\beta} r(t) x^{\prime \prime}(t)^{2} d t \leq & 4 A_{1}^{2}(\alpha, \beta) \int_{\alpha}^{\beta} r(t) x^{\prime \prime}(t)^{2} d t \\
& +G_{1}\left(\alpha, \beta, M_{1,1}\right) \int_{\alpha}^{\beta}|r(t)|\left|x^{\prime \prime}(t)^{2}\right| d t+ \\
& H_{1}\left(\alpha, \beta, G_{1,0}\right) \int_{\alpha}^{\beta} r(t) x^{\prime \prime}(t)^{2} d t
\end{aligned}
$$

Cancelling the term $\int_{\alpha}^{\beta}|r(t)|\left|x^{\prime \prime}(t)^{2}\right| d t$, $4 \mathrm{~A}_{1}^{2}(\alpha, \beta)+H_{1}\left(\alpha, \beta, G_{1,0}\right)+M_{1}\left(\alpha, \beta, G_{1,1}\right) \geq 1$,

The proof of (3.10) is similar to (3.9) by replaceing $H_{1}\left(\alpha, \beta, G_{1,0}\right)$ by $H_{2}\left(\alpha, \beta, G_{1,0}\right)$ replacing $A_{1}(\alpha, \beta)$ by $A_{2}(\alpha, \beta)$ and replaceing $M_{1}\left(\alpha, \beta, G_{1,1}\right)$ by $M_{1}\left(\alpha, \beta, G_{2,1}\right)$. The proof is complete.

In the following, we apply the Clark and Hinton inequality (2.15) whith $r=2$ to get a new result. 
Theorem 3.2. Assume that $r(t)$ is a non-increasing function and suppose that $x(t)$ is a solution of (1.5). If $x(\alpha)=x^{\prime}(\alpha)=x^{\prime}(\beta)=0$, then

$$
4 A_{1}^{2}(\alpha, \beta)+\frac{(\beta-\alpha)^{\frac{3}{2}}}{\sqrt{3} r(\beta)}\left(\int_{\alpha}^{\beta}\left|Q_{1}(t)\right|^{2} d t\right)^{\frac{1}{2}} \geq 1,
$$

where $Q_{1}(t)$ and $A_{1}(\alpha, \beta)$ are defined as in (3.1) and (3.5), respectively.

Proof. Multiplying (1.5) by $x^{\prime}(t)$ and proceeding as in the proof of Theorem (3.1) to get,

$$
\begin{aligned}
\int_{\alpha}^{\beta}|r(t)|\left|x^{\prime \prime}(t)\right|^{2} d t & \leq \int_{\alpha}^{\beta}\left|R_{1}(t)\right|\left|x^{\prime}(t)\right|^{2} d t+\int_{\alpha}^{\beta}\left|Q_{1}(t)\right||x(t)|\left|x^{\prime \prime}(t)\right| d t \\
& +\int_{\alpha}^{\beta}|u(t)|\left|x^{\prime}(t)\right|\left|x^{\prime \prime}(t)\right| d t .
\end{aligned}
$$

where $\left|R_{1}(t)\right|=\left|Q_{1}(t)\right|+|p(t)|$ and $u(t)=r^{\prime}(t)$.

Applying the Schwarz inequality

$$
\int_{\alpha}^{\beta} f(t) g(t) d t \leq\left(\int_{\alpha}^{\beta} f(t)^{2} d t\right)^{\frac{1}{2}}\left(\int_{\alpha}^{\beta} g(t)^{2} d t\right)^{\frac{1}{2}},
$$

on the integral $\int_{\alpha}^{\beta}\left|Q_{1}(t)\right||x(t)|\left|x^{\prime \prime}(t)\right| d t$, we have that

$$
\int_{\alpha}^{\beta}\left|Q_{1}(t)\right||x(t)|\left|x^{\prime \prime}(t)\right| d t \leq\left(\int_{\alpha}^{\beta}\left|Q_{1}(t)\right|^{2} d t\right)^{\frac{1}{2}}\left(\int_{\alpha}^{\beta}|x(t)|^{2}\left|x^{\prime \prime}(t)\right|^{2} d t\right)^{\frac{1}{2}} .
$$

Applying the inequality (2.15) and using the assumpution $x(\alpha)=x^{\prime}(\alpha)$, we get that

$$
\left(\int_{\alpha}^{\beta}|x(t)|^{2}\left|x^{\prime \prime}(t)\right|^{2} d t\right)^{\frac{1}{2}} \leq \frac{(\beta-\alpha)^{\frac{3}{2}}}{\sqrt{3}} \int_{\alpha}^{\beta}\left|x^{\prime \prime}(t)\right|^{2} d t .
$$

Substituting (3.24) into (3.23) and using the assumpution that $r(t)$ is a non-increasing function, we have

$$
\int_{\alpha}^{\beta}\left|Q_{1}(t)\right||x(t)|\left|x^{\prime \prime}(t)\right| d t \leq \frac{(\beta-\alpha)^{\frac{3}{2}}}{\sqrt{3} r(\beta)}\left(\int_{\alpha}^{\beta}\left|Q_{1}(t)\right|^{2} d t\right)^{\frac{1}{2}} \int_{\alpha}^{\beta} r(t)\left|x^{\prime \prime}(t)\right|^{2} d t .
$$


Applying Hardy's inequality (2.1) on the integral $\int_{\alpha}^{\beta}\left|R_{1}(t)\right|\left|x^{\prime}(t)\right|^{2} d t$, we obtain

$$
\int_{\alpha}^{\beta}\left|R_{1}(t)\right|\left|x^{\prime}(t)\right|^{2} d t \leq 4 A_{1}^{2}(\alpha, \beta) \int_{\alpha}^{\beta}|r(t)|\left|x^{\prime \prime}(t)\right|^{2} d t
$$

where $x^{\prime}(\alpha)=x^{\prime}(\beta)=0$. Substituting (3.25) and (3.26) into (3.21), we obtain

$$
\begin{aligned}
\int_{\alpha}^{\beta} r(t) x^{\prime \prime}(t)^{2} d t \leq & 4 A_{1}^{2}(\alpha, \beta) \int_{\alpha}^{\beta} r(t) x^{\prime \prime}(t)^{2} d t \\
& +\frac{(\beta-\alpha)^{\frac{3}{2}}}{\sqrt{3} r(\beta)}\left(\int_{\alpha}^{\beta} Q_{1}(t)^{2} d t\right)^{\frac{1}{2}} \int_{\alpha}^{\beta} r(t) x^{\prime \prime}(t)^{2} d t .
\end{aligned}
$$

The desired inequality (3.20) followed by cancelling the term $\int_{\alpha}^{\beta} r(t) x^{\prime \prime}(t)^{2} d t$. The proof is complete.

Remark 3.1. When $r(t)=1, p(t)=0$ in Theorem 3.2, we have the result in ([21] corollary 6$)$.

Now, we will prove some new result when $r(t)=1$.

Theorem 3.3. Suppose that $r(t)=1$ and asumme that $x(t)$ is a solution of (1.5). If $x(\alpha)=x^{\prime}(\alpha)=x^{\prime}(\beta)=0$, or $x(\beta)=x^{\prime}(\beta)=x^{\prime}(\alpha)=0$, then

$$
P \frac{\pi(\beta-\alpha)^{2}}{16}+Q \frac{(\beta-\alpha)^{3}}{6} \geq 1,
$$

where $P(t)=\max _{\alpha \leq t \leq \beta}|p(t)|$, and $Q(t)=\max _{\alpha \leq t \leq \beta}|q(t)|$.

Proof. The proof is similar the proof of (theorem 5 [21])

As a special case when $p(t)=0$ in Theorem 3.3, we have the following result.

Corollary 3.1. Suppose that $r(t)=1, p(t)=0$ and let $x(t)$ be a nontrivial solution of (1.5). If $x(\alpha)=x^{\prime}(\alpha)=x^{\prime}(\beta)=0$, then

$$
\max _{\alpha \leq t \leq \beta}|q(t)| \geq \frac{6}{(\beta-\alpha)^{3}} .
$$


Corollary 3.2. Suppose that $r(t)=1, q(t)=0$ and let $x(t)$ be a nontrivial solution of (1.5). If $x(\alpha)=x^{\prime}(\alpha)=x^{\prime}(\beta)=0$, then

$\max _{\alpha \leq t \leq \beta}|p(t)| \geq \frac{16}{\pi(\beta-\alpha)^{2}}$.

In the following, we apply an inequality due to Boyd [4] to obtain new results. The Boyd inequality states that: If $y \in C^{1}[\alpha, \beta]$ with $y(\alpha)=0$ (or $y(\beta)=0)$, then

$$
\int_{\alpha}^{\beta}|y(t)|^{\nu}\left|y^{\prime}(t)\right|^{\eta} d t \leq N(\nu, \eta, s)(\beta-\alpha)^{\nu}\left[\int_{a}^{b}\left|y^{\prime}(t)\right|^{s} d t\right]^{\frac{\nu+\eta}{s}}
$$

where $\nu>0, s>1,0 \leq \eta<s$,

$$
\begin{gathered}
N(\nu, \eta, s):=\frac{(s-\eta) \nu^{\nu} r^{\nu+\eta-s}}{(s-1)(\nu+\eta)(I(\nu, \eta, s))^{\nu}} \\
r:=\left\{\frac{\nu(s-1)+(s-\eta)}{(s-1)(\nu+\eta)}\right\}^{\frac{1}{s}}
\end{gathered}
$$

and

$$
\mathrm{I}(\nu, \eta, s):=\int_{0}^{1}\left\{1+\frac{s(\eta-1)}{s-\eta} t\right\}^{-(\nu+\eta+s \nu) / s \nu}[1+(\eta-1)] t^{\frac{1}{\nu-1}} d t .
$$

Note that an inequality of type (3.29) also holds when $y(\alpha)=y(\beta)=0$. Choose $c=(\alpha+\beta) / 2$ and apply (3.29) to $[\alpha, c]$ and $[c, \beta]$, we obtain

$$
(3.31) \int_{\alpha}^{\beta}|y(t)|^{\nu}\left|y^{\prime}(t)\right|^{\eta} d t \leq N(\nu, \eta, s)\left(\frac{\beta-\alpha}{2}\right)^{\nu}\left[\int_{\alpha}^{\beta}\left|y^{\prime}(t)\right|^{s} d t\right]^{\frac{\nu+\eta}{s}},
$$

where $N(\nu, \eta, s)$ is defined as in (3.30). An inequality of type (3.29) holds when $\eta=s$ and $y(\alpha)=0$ (or $y(\beta)=0)$. In this case, inequality (3.29) becomes

$$
\int_{\alpha}^{\beta}|y(t)|^{\nu}\left|y^{\prime}(t)\right|^{\eta} d t \leq L(\nu, \eta)(\beta-\alpha)^{\nu}\left[\int_{\alpha}^{\beta}\left|y^{\prime}(t)\right|^{\eta} d t\right]^{\frac{\nu+\eta}{\eta}},
$$

where

$$
L(\nu, \eta):=\frac{\eta \nu^{\eta}}{\nu+\eta}\left(\frac{\nu}{\nu+\eta}\right)^{\frac{\nu}{\eta}}\left(\frac{\Gamma\left(\frac{\eta+1}{\eta}+\frac{1}{\nu}\right)}{\Gamma\left(\frac{\eta+1}{\eta}\right) \Gamma\left(\frac{1}{\nu}\right)}\right)^{\nu},
$$

and $\Gamma$ is the gamma function. 
Theorem 3.4. Assume that $r(t)$ is non-incresing function and $x(t)$ is a nontrivial solution of (1.5).

(1) If $x(\alpha)=x^{\prime}(\alpha)=x^{\prime}(\beta)=0$, then

$$
4 B_{1}^{2}(\alpha, \beta)+\frac{8(\beta-\alpha)}{\pi r(\beta)} \psi^{2}(\alpha, \beta)\left(\int_{\alpha}^{\beta}|q(t)|^{2} d t\right)^{\frac{1}{2}}+Z_{1}\left(\alpha, \beta, G_{1,1}\right) \geq 1 .
$$

(2) If $x(\beta)=x^{\prime}(\beta)=x(\alpha)=0$, then

$$
4 B_{2}^{2}(\alpha, \beta)+\frac{8(\beta-\alpha)}{\pi r(\beta)} \psi^{2}(\alpha, \beta)\left(\int_{\alpha}^{\beta}|q(t)|^{2} d t\right)^{\frac{1}{2}}+G_{1}\left(\alpha, \beta, G_{2,1}\right) \geq 1 .
$$

Proof. To prove (3.34), we multiply (1.5) by $x^{\prime}(t)$ and integrating from $\alpha$ to $\beta$, we obtain

$\int_{\alpha}^{\beta} x^{\prime}(t)\left(r(t)\left(x^{\prime}(t)\right)\right)^{\prime \prime} d t=-\int_{\alpha}^{\beta} p(t)\left(x^{\prime}(t)\right)^{2} d t-\int_{\alpha}^{\beta} q(t) x(t) x^{\prime}(t) d t$. (3.36)

Integrating by parts the left hand side, and using the assumption that $x(\alpha)=x^{\prime}(\alpha)=x^{\prime}(\beta)=0$, we get that

$$
\int_{\alpha}^{\beta} x^{\prime}\left(r\left(x^{\prime}\right)\right)^{\prime \prime} d t=-\int_{\alpha}^{\beta} r^{\prime}(t) x^{\prime}(t) x^{\prime \prime}(t) d t-\int_{\alpha}^{\beta} r(t)\left(x^{\prime \prime}(t)\right)^{2} d t
$$


By substitutian (3.36) into (3.37), we give

$$
\begin{gathered}
{ }_{\alpha}^{\beta}|r(t)|\left|x^{\prime \prime}(t)\right|^{2} d t \leq \\
\int_{\alpha}^{\beta}|p(t)|\left|x^{\prime}(t)\right|^{2} d t+\int_{\alpha}^{\beta}|q(t)||x(t)|\left|x^{\prime}(t)\right| d t \\
+\int_{\alpha}^{\beta}|u(t)|\left|x^{\prime}(t)\right|\left|x^{\prime \prime}(t)\right| d t, u(t)=r^{\prime}(t) .
\end{gathered}
$$

Applying the inequality (2.1) on the integral $\int_{\alpha}^{\beta}|p(t)|\left|x^{\prime}(t)\right|^{2} d t$ with $m=n=2$, we get

$$
\int_{\alpha}^{\beta}|p(t)|\left|x^{\prime}(t)\right|^{2} d t \leq 4 \psi^{2}(\alpha, \beta) \int_{\alpha}^{\beta}|r(t)|\left|x^{\prime \prime}(t)^{2}\right| d t
$$

where $\psi(\alpha, \beta)$ is defined as in (3.8).

Applying Schwarz inequality (3.22) on $\int_{\alpha}^{\beta}|q(t)||x(t)|\left|x^{\prime}(t)\right| d t$, to get

$$
\int_{\alpha}^{\beta}|q(t)||x(t)|\left|x^{\prime}(t)\right| d t \leq\left(\int_{\alpha}^{\beta}|q(t)|^{2} d t\right)^{\frac{1}{2}}\left(\int_{\alpha}^{\beta}|x(t)|^{2}\left|x^{\prime}(t)\right|^{2} d t\right)^{\frac{1}{2}} .
$$

Now, by applying the inequality (3.32) on the integral $\int_{\alpha}^{\beta}|x(t)|^{2}\left|x(t)^{\prime}\right|^{2} d t$ with $\nu=\eta=2$. we obtain

$$
\int_{\alpha}^{\beta}|x|^{2}\left|x^{\prime}\right|^{2} d t \leq \frac{4(\beta-\alpha)^{2}}{\pi^{2} r^{2}(\beta)}\left[\int_{\alpha}^{\beta} r(t)\left|x^{\prime}\right|^{2} d t\right]^{2},
$$

where $r(t)$ is a non-increasing function. Substituting (3.41) into (3.40), we get

$$
\int_{\alpha}^{\beta}|q(t)||x(t)|\left|x^{\prime}(t)\right| d t \leq\left(\int_{\alpha}^{\beta}|q(t)|^{2} d t\right)^{\frac{1}{2}} \frac{2(\beta-\alpha)}{\pi r(\beta)} \int_{\alpha}^{\beta} r(t)\left|x^{\prime}\right|^{2} d t .
$$


Applying the inequality (2.1) on the integral $\int_{\alpha}^{\beta} r(t)\left|x^{\prime}\right|^{2} d t$ with $m=$ $n=2$, we get

$$
\int_{\alpha}^{\beta}|r(t)|\left|x^{\prime}(t)\right|^{2} d t \leq 4 B_{1}^{2}(\alpha, \beta) \int_{\alpha}^{\beta}|r(t)|\left|x^{\prime \prime}(t)\right|^{2} d t .
$$

Substituting (3.43) into (3.42), we have

$$
\int_{\alpha}^{\beta}|q(t)||x(t)|\left|x^{\prime}(t)\right| d t \leq\left(\int_{\alpha}^{\beta}|q(t)|^{2} d t\right)^{\frac{1}{2}} \frac{8(\beta-\alpha)}{\pi r(\beta)} \psi^{2}(\alpha, \beta) \int_{\alpha}^{\beta}|r(t)|\left|x^{\prime \prime}(t)\right|^{2} d t .
$$

Applying the inequality (2.8) on the integral

$$
\int_{\alpha}^{\beta}|u(t)|\left|x^{\prime}(t)\right|\left|x^{\prime \prime}(t)\right| d t,
$$

we obtain

$$
\int_{\alpha}^{\beta}|u(t)|\left|x^{\prime}(t)\right|\left|x^{\prime \prime}(t)\right| d t \leq Z_{1}\left(\alpha, \beta, G_{1,1}\right) \int_{\alpha}^{\beta}|r(t)|\left|x^{\prime \prime}(t)^{2}\right| d t
$$

where $Z_{1}\left(\alpha, \beta, G_{1,1}\right)$ is defined in (3.3).

Substituting (3.39), (3.44) and (3.45) into (3.38), we obtain

$$
\begin{aligned}
\int_{\alpha}^{\beta}|r(t)|\left|x^{\prime \prime}(t)\right|^{2} d t \leq & 4 B_{1}^{2}(\alpha, \beta) \int_{\alpha}^{\beta}|r(t)|\left|x^{\prime \prime}(t)\right|^{2} d t \\
& +\left(\int_{\alpha}^{\beta}|q(t)|^{2} d t\right)^{\frac{1}{2}} \frac{8(\beta-\alpha)}{\pi r(\beta)} \psi^{2}(\alpha, \beta) \int_{\alpha}^{\beta}|r(t)|\left|x^{\prime \prime}(t)\right|^{2} d t \\
& +Z_{1}\left(\alpha, \beta, G_{1,1}\right) \int_{\alpha}^{\beta}|r(t)|\left|x^{\prime \prime}(t)^{2}\right| d t .
\end{aligned}
$$

Cancelling the term $\int_{\alpha}^{\beta}|r(t)|\left|x^{\prime \prime}(t)\right|^{2} d t$, we obtain

$4 \mathrm{~B}_{1}^{2}(\alpha, \beta)+\frac{8(\beta-\alpha)}{\pi r(\beta)} \psi^{2}(\alpha, \beta)\left(\int_{\alpha}^{\beta}|q(t)|^{2} d t\right)^{\frac{1}{2}}+Z_{1}\left(\alpha, \beta, G_{1,1}\right) \geq 1$,

which is the desired inequality (3.34). In order to prove (3.35) we proceed as the proof of $(3.34)$ and using $B_{2}(\alpha, \beta)$ instead of $B_{1}(\alpha, \beta)$ and replaceing $Z_{1}\left(\alpha, \beta, G_{1,1}\right)$ by $Z_{2}\left(\alpha, \beta, G_{2,1}\right)$. Hence, the proof is complete.

In the following, we present some results related to the boundary conditions $(i i)$. 
Theorem 3.5. Assume that $x(t)$ is a nontrivial solution of (1.5).

(1). If $x^{\prime}(\alpha)=x^{\prime}(\beta)=x^{\prime \prime}(\beta)=0$, then

$$
4 B_{1}^{2}(\alpha, \beta)+C_{\alpha}+Z_{1}\left(\alpha, \beta, G_{1,1}\right) \geq 1 .
$$

(2) If $x^{\prime}(\beta)=x^{\prime}(\alpha)=x^{\prime \prime}(\alpha)=0$, then

$$
4 B_{2}^{2}(\alpha, \beta)+C_{\beta}+G_{1}\left(\alpha, \beta, G_{2,1}\right) \geq 1 .
$$

Proof. To prove (3.46), we multiply (1.5) by $x^{\prime}(t)$ and integrating from $\alpha$ to $\beta$, we obtain

$\int_{\alpha}^{\beta} x^{\prime}(t)\left(r(t)\left(x^{\prime}(t)\right)\right)^{\prime \prime} d t=-\int_{\alpha}^{\beta} p(t)\left(x^{\prime}(t)\right)^{2} d t-\int_{\alpha}^{\beta} q(t) x(t) x^{\prime}(t) d t$. $(3.48)$

Integrating by parts the left hand side, we get that

$$
\int_{\alpha}^{\beta} x^{\prime}\left(r\left(x^{\prime}\right)\right)^{\prime \prime} d t=\left.x^{\prime}(t)\left(r\left(x^{\prime}\right)\right)^{\prime}\right|_{\alpha} ^{\beta}-\int_{\alpha}^{\beta} r^{\prime}(t) x^{\prime}(t) x^{\prime \prime}(t) d t-\int_{\alpha}^{\beta} r(t)\left(x^{\prime \prime}(t)\right)^{2} d t \text {. }
$$

Using the assumption that $x^{\prime}(\alpha)=x^{\prime}(\beta)=x^{\prime \prime}(\beta)=0$, we have

$$
\int_{\alpha}^{\beta} x^{\prime}\left(r\left(x^{\prime}\right)\right)^{\prime \prime} d t=-\int_{\alpha}^{\beta} r^{\prime}(t) x^{\prime}(t) x^{\prime \prime}(t) d t-\int_{\alpha}^{\beta} r(t)\left(x^{\prime \prime}(t)\right)^{2} d t
$$

By substitutian (3.49) into (3.48), we obtain

$$
\begin{gathered}
{ }_{\alpha}^{\beta}|r(t)|\left|x^{\prime \prime}(t)\right|^{2} d t \leq \\
\int_{\alpha}^{\beta}|p(t)|\left|x^{\prime}(t)\right|^{2} d t+\int_{\alpha}^{\beta}|q(t)||x(t)|\left|x^{\prime}(t)\right| d t \\
+\int_{\alpha}^{\beta}|u(t)|\left|x^{\prime}(t)\right|\left|x^{\prime \prime}(t)\right| d t,
\end{gathered}
$$

where $u(t)=r^{\prime}(t)$

Applying the inequality (2.1) on the integral $\int_{\alpha}^{\beta}|p(t)|\left|x^{\prime}(t)\right|^{2} d t$ with $n=m=2$, we get

$$
\int_{\alpha}^{\beta}|p(t)|\left|x^{\prime}(t)\right|^{2} d t \leq 4 B_{1}^{2}(\alpha, \beta) \int_{\alpha}^{\beta}|r(t)|\left|x^{\prime \prime}(t)^{2}\right| d t,
$$


where $B_{1}(\alpha, \beta)$ is deffined as in (3.6). Agin applying the inequality (2.11) on the integral $\int_{\alpha}^{\beta}|q(t)||x(t)|\left|x^{\prime}(t)\right| d t$, we get

$$
\int_{\alpha}^{\beta}|q(t)||x(t)|\left|x^{\prime}(t)\right| d t \leq C_{\alpha} \int_{\alpha}^{\beta}|r(t)|\left|x^{\prime \prime}(t)^{2}\right| d t,
$$

where $C_{\alpha}$ is defined as in (3.6). Applying the inequality (2.8) on the integral $\int_{\alpha}^{\beta}|u(t)|\left|x^{\prime}(t)\right|\left|x^{\prime \prime}(t)\right| d t$

we obtaine

$$
\int_{\alpha}^{\beta}|u(t)|\left|x^{\prime}(t)\right|\left|x^{\prime \prime}(t)\right| d t \leq Z_{1}\left(\alpha, \beta, G_{1,1}\right) \int_{\alpha}^{\beta}|r(t)|\left|x^{\prime \prime}(t)^{2}\right| d t
$$

where $Z_{1}\left(\alpha, \beta, G_{1,1}\right)$ is defined in (3.3).

Substituting (3.51), (3.52) and (3.53) into (3.50), we obtain

$$
\begin{aligned}
\int_{\alpha}^{\beta}|r(t)|\left|x^{\prime \prime}(t)\right|^{2} d t \leq & 4 B_{1}^{2} \int_{\alpha}^{\beta}|r(t)|\left|x^{\prime \prime}(t)^{2}\right| d t+C_{\alpha} \int_{\alpha}^{\beta}|r(t)|\left|x^{\prime \prime}(t)^{2}\right| d t \\
& +Z_{1}\left(\alpha, \beta, G_{1,1}\right) \int_{\alpha}^{\beta}|r(t)|\left|x^{\prime \prime}(t)^{2}\right| d t .
\end{aligned}
$$

By cancelling the term $\int_{\alpha}^{\beta}|r(t)|\left|x^{\prime \prime}(t)^{2}\right| d t$, we get that

$4 \mathrm{~B}_{1}^{2}(\alpha, \beta)+C_{\alpha}++Z_{1}\left(\alpha, \beta, G_{1,1}\right) \geq 1$

which is the desired result (3.46). In order to prove (3.47) we proceed as the proof of (3.46) and using the inequality $B_{2}(\alpha, \beta)$ instead of $B_{1}(\alpha, \beta)$, replacing $C_{\alpha}$ and $Z_{1}\left(\alpha, \beta, G_{1,1}\right)$ by $C_{\beta}$ and $Z_{2}\left(\alpha, \beta, G_{2,1}\right)$, respectively. Hence, the proof is complete.

\section{References}

[1] R. P. Agarwal and P. R. Krinamoorthy, "On the uniqueness of solutions of nonlinear boundary value problems", Journal of Mathematical Physics, vol. 10, pp. 17-31, 1976.

[2] R. P. Agarwal and P. Pang, Opial Inequalities with Applications in Differential and Difference Equations. Dordrecht: Kluwer Academic, 1995. 
[3] P. R. Beesak and K. M. Das, "Extensions of Opial's inequality”, Pacific Journal of Mathematics, vol. 26, pp. 215-232, 1968.

[4] D. Boyd, "Best constants in a class of integral inequalities", Pacific Journal of Mathematics, vol. 30, pp. 367-383, 1969.

[5] I. Brnetić and J. Pečarić, "Some new Opial-type inequalities", Mathematical Inequalities \& Applications, vol. 1, no. 3, pp. 385-390, 1998.

[6] G. Casadei, "Sul teorema di unicità di De La Vallée Poussin per equazioni differenziali del terzo ordine", Rendiconti del Seminario Matematico della Università di Padova, vol. 41, pp. 300-315, 1968.

[7] S. Clark and D. Hinton, "Some disconjugacy criteria for differential equations with oscillatory coefficients", Mathematische Nachrichten, vol. 287, no. 12-13, pp. 1476- 1489, 2005.

[8] A. M. Fink, "On Opial's inequality for $f^{(n)}$ ", Proceedings of the American Mathematical Society, vol. 155, pp. 177-181, 1992.

[9] A. Kufner and L.-E. Persson, Weighted Inequalities of Hardy Type. River Edge, NJ: World Scientific, 2003.

[10] A. Kufner, L. Maligranda, and L. Persson, The Hardy Inequalities: About Its History and Some Related Results. Pilsen: Vydavatelský servis, 2007.

[11] A. Lasota, "Sur la distance entre les zéros de l'équation différentielle linéaire du troisième ordre", Annales Polonici Mathematici, vol. 13, pp. 129-132, 1963.

[12] A. M. Liapounoff, "Problème général de la stabilité du mouvement", Annales de la Faculté des sciences de Toulouse: Mathématiques, vol. 9, pp. 2037-474, 1907.

[13] R. M. Mathsen, "A Disconjugacy condition for $y^{\prime \prime \prime}+a_{2} y^{\prime \prime}+a_{1} y^{\prime}+a_{0} y=$ 0", Proceedings of the American Mathematical Society, pp. 627-632, 1966.

[14] O. Niccoletti, "Sulle consizioni iniziali che determinano gli integrale delle equazioni differenziali ordinarie", Atti della Accademia delle Scienze di Torino, vol. 33, pp. 746-759, 1897. 
[15] E. Picard, "Mémoire sur la théorie des équations aux dérivées partielleset la méthode des approximations successives", Journal de Mathématiques Pures et Appliquées, vol. 6, pp. 145-210, 231, 1890.

[16] E. Picard, "Sur l'application des méthodes d'approximations successives à l'étude de certaines équations différentielles ordinaires", Journal de Mathématiques Pures et Appliquées, vol. 9, pp. 217-271, 1893.

[17] U. Richard, "Metodi diversi per ottenere diseguaglianze alla De La Vallée Poussin nelle equazioni differenziali ordinarie del secondo e terzo ordine", Rendiconti del Seminario Matematico dell' Università e del Politecnico di Torino, vol. 27, pp. 35- 68, 1967-1968.

[18] S. H. Saker, B., Sudha, M. A. Arahet, and E., Thandapani, "Distribution of zeros of second order superlinear and sublinear neutral delay differential equations", Revista de la Real Academia de Ciencias Exactas, Físicas y Naturales. Serie A. Matemáticas, vol. 113, 2018.

[19] S. H. Saker, "Applications of Opial and Wirtinger inequalities on zeros of third order differential equations", Dynamic Systems and Applications, vol. 20, no. 4, pp. 479- 494, 2011.

[20] S. H. Saker, "Lyapunov type inequalities for a second order differential equations with a damping term", Annales Polonici Mathematici, vol. 103, pp. 37-57, 2012.

[21] S. H. Saker and M. A. Arahet, "Distributions of Zeros of Solutions for Third-Order Differential Equations with Variable Coefficients", Mathematical Problems in Engineering, vol. 2015, Art. ID 158460, 2015.

[22] C. de la Vallée Poussin, "Sur l'équation différentielle linéaire du second ordre. Détermination d'une intégrale par deux valeurs assignées. Extension aux équations d'ordre n", Journal de Mathématiques Pures et Appliquées, vol. 8, pp. 125-144, 1929.

[23] X. Yang, "On Liapunov-type inequality for a certain higher-order differential equations", Applied Mathematics and Computation, vol. 134, no. 2-3, pp. 307-317, 2003. 


\title{
C. Cesarano
}

Section of Mathematics,

International Telematic University Uninettuno,

CorsoVittorio Emanuele II,

39, 00186 Roma,

Italy

e-mail: c.cesarano@uninettunouniversity.net

Corresponding author

\author{
M. A. Arahet \\ Department of Mathematics, \\ Faculty of Science, \\ Amran University, \\ Yemen \\ e-mail: malroheet@yahoo.com \\ and
}

\section{T. M. Al-shami}

Department of Mathematics,

Faculty of Science,

Sana'a University,

Yemen

e-mail:tareqalshami83@gmail.com 